\title{
TEMPERATURES IN SEVERAL VARIABLES: KERNEL FUNCTIONS, REPRESENTATIONS, AND PARABOLIC BOUNDARY VALUES
}

\author{
BY \\ JOHN T. KEMPER
}

\begin{abstract}
This work develops the notion of a kernel function for the heat equation in certain regions of $n+1$-dimensional Euclidean space and applies that notion to the study of the boundary behavior of nonnegative temperatures. The regions in question are bounded between spacelike hyperplanes and satisfy a parabolic Lipschitz condition at points on the lateral boundary.

Kernel functions (normalized, nonnegative temperatures which vanish on the parabolic boundary except at a single point) are shown to exist uniquely. A representation theorem for nonnegative temperatures is obtained and used to establish the existence of finite parabolic limits at the boundary (except for a set of heat-related measure zero).
\end{abstract}

0 . Introduction. The notion of a kernel function has been developed in the case of the Laplace operator by Hunt and Wheeden [4], who prove existence and uniqueness for such functions in Lipschitz domains of $n$-dimensional Euclidean space $\boldsymbol{R}^{n}$ and use these functions to study the nontangential boundary behavior of harmonic functions which have a one-sided bound in the domain. In [7] we reported analogous results for the heat equation in certain regions of the plane. These results had been obtained in [6]. There, difficulties in the application of the techniques of [4] to the heat equation were overcome and some simplification of those techniques was achieved, notably in the proof of uniqueness of kernel functions. However, technical problems prevented treatment of the heat equation in more than one space dimension. The present work extends those results to the case of several space variables.

In $\S 1$ existence and uniqueness of kernel functions is established and a representation theorem is obtained for temperatures with a one-sided bound in a region satisfying a certain mixed-Lipschitz condition (conditions L1 and L2 below). The main result of $\S 2$ is the existence almost everywhere (with respect to caloric measure) on the parabolic boundary of finite parabolic limits for temperatures with a one-sided bound in the region. This generalizes the work of Jones and Tu [5] and Hattemer [2], who considered regions less general than those dealt with here.

Received by the editors March 22, 1971.

AMS 1970 subject classifications. Primary 35K05; Secondary 35C15.

Key words and phrases. Heat equation, kernel function, parabolic limit.

Copyright (C) 1972, American Mathematical Society 
1. Kernel functions and a representation theorem. We denote by $(x, t)$ a point in $\boldsymbol{R}^{n+1}$, where $x=\left(x_{1}, x_{2}, \ldots, x_{n}\right)=\left(x^{\prime}, x_{n}\right)$ are the space variables and $t$ the time variable. For a domain $D$ in $R^{n+1}$, we let $\partial_{p} D$ be the parabolic boundary of $D$; i.e. $\partial_{p} D$ is the set of points on the boundary of $D$ which can be connected to some interior point of $D$ by a closed curve having strictly increasing $t$-coordinate. $D$ is a regular domain for the Dirichlet problem for the heat equation if that problem is solvable in the Weiner-Perron sense for any (Borel) integrable boundary values. For regular domains we have the following:

Definition. If $(x, t) \in D$ and $Z \subset \partial D$ is a Borel measurable set, the caloric measure at $(x, t)$ of $Z$, denoted $\omega_{D}^{(x, t)}(Z)$, is the value at $(x, t)$ of the unique solution of the Dirichlet problem for the heat equation in $D$ with boundary data given by the characteristic function of $Z$. (When there is no possibility of confusion, we shall suppress the subscript $D$, writing $\omega^{(x, t)}(Z)$.)

Throughout our discussion $(X, T)$ will denote a fixed point in $D$. If $t \leqq$ $\inf \{s: \exists y$ with $(y, s) \in Z\}$, we must have $\omega^{(x, t)}(Z)=0$ by the maximum principle. Furthermore, if $(x, t) \in D$ can be joined to $(X, T)$ by a closed curve in $D$ with strictly increasing $t$-coordinate, then Harnack's inequality and Besicovitch's general theory of differentiation [1] imply that the Radon-Nikodym derivative, $\partial \omega^{(x, t)} / \partial \omega^{(X, T)}$, which exists in $L^{1}\left(\omega^{(X, T)}\right)$, is given a.e. $\left(\omega^{(X, T)}\right)$ by

$$
\operatorname{limit}_{\Delta_{n} \rightarrow(y, s)} \frac{\omega^{(x, t)}\left(\Delta_{n}\right)}{\omega^{(X, T)}\left(\Delta_{n}\right)},
$$

where $\Delta_{n}$ is any sequence of closed sets in $\partial_{p} D$ which contain $(y, s)$ and satisfy $\inf _{n}\left(\omega^{(X, T)}\left(\Delta_{n}\right) / \omega^{(X, T)}\left(B_{n}\right)\right)>0$, where $B_{n}$ is the intersection of $\partial_{p} D$ with the smallest sphere centered at $(y, s)$ and containing $\Delta_{n}$.

We will be concerned with the following concept of a kernel function:

Definition. If $(Y, S) \in \partial_{p} D$ with $S<T$, a function $K(x, t)$ defined in $D$ is a kernel function at $(Y, S)$ for the heat equation in $D$ with respect to $(X, T)$ if

(i) $K(x, t) \geqq 0$ for $(x, t) \in D$,

(ii) $K(x, t)$ satisfies the heat equation, $\Delta K=K_{t}$, in $D$,

(iii) $\operatorname{limit}_{(x, t) \rightarrow(y, s) ;(x, t) \in D} K(x, t)=0$ for $(y, s) \in \partial_{p} D-\{(Y, S)\}$,

(iv) $K(X, T)=1$ (normalization condition).

(If $S \geqq T$, we shall take the kernel function at $(Y, S)$ with respect to $(X, T)$ to be identically zero.)

Suppose that $D$ is a regular domain for the heat equation, and let $D_{T}$ $=D \cap\{(x, t): t \leqq T\}$. It is clear that if $S<T$ and a function $K(x, t)$ satisfies (i)-(iv) in $D_{T}$, then $K(x, t)$ can be extended to a kernel function in $D$ by solving a Dirichlet problem in $D-D_{S+\varepsilon}$. Conversely, if $K(x, t)$ is a kernel function at $(Y, S)$ in $D$, then its restriction to $D_{T}$ is a kernel function at $(Y, S)$ in $D_{T}$.

To obtain existence and uniqueness of kernel functions at a point $(Y, S) \in \partial_{p} D$, we require additional restrictions on $\partial_{p} D$ in a neighborhood of $(Y, S)$. We allow two conditions: 
Condition L1. $\partial_{p} D$ is given locally at $(Y, S)$ by $t=S$.

Condition L2. $\partial_{p} D$ is given locally at $(Y, S)$ by a function satisfying a mixedLipschitz condition with exponent 1 in the space variables and $\frac{1}{2}$ in the time variable.

Specifically, for condition L2 to hold, there is a sphere $\mathcal{O}$ with center $(Y, S)$, local space coordinates $x$, and a function $f\left(x^{\prime}, t\right)$ defined in $\mathcal{O}^{\prime}=\left\{\left(x^{\prime}, t\right): \exists x_{n}\right.$ with $\left.\left(x^{\prime}, x_{n}, t\right) \in \mathcal{O}\right\}$ satisfying

$$
\left|f\left(x^{\prime}, t\right)-f\left(x_{0}^{\prime}, t_{0}\right)\right| \leqq C\left(\left|x^{\prime}-x_{0}^{\prime}\right|+\left|t-t_{0}\right|^{1 / 2}\right)
$$

for $\left(x^{\prime}, t\right)$ and $\left(x_{0}^{\prime}, t_{0}\right)$ in $\mathcal{O}^{\prime}$, such that

$$
\begin{aligned}
& \mathcal{O} \cap \partial_{p} D \cap\{(x, t): t>S\}=\mathcal{O} \cap\left\{(x, t): x_{n}=f\left(x^{\prime}, t\right)\right\} \cap\{t>S\}, \\
& \mathcal{O} \cap D \cap\{(x, t): t>S\}=\mathcal{O} \cap\left\{(x, t): x_{n}>f\left(x^{\prime}, t\right)\right\} \cap\{t>S\} .
\end{aligned}
$$

Thus, $\mathrm{L} 2$ describes the "side" points of $\partial_{p} D$. Similarly, condition L1 describes the "bottom" points of $\partial_{p} D$. In the case of a "bottom corner" point $(Y, S)$, condition L2 applies. In fact, by extending the domain, we may assume that $\partial_{p} D$ is given by the mixed-Lipschitz function $f\left(x^{\prime}, t\right)$ in a complete neighborhood of $(Y, S)$. We also note that any point $(Y, S) \in \partial_{p} D$ satisfying either condition L1 or L2 is a regular boundary point for the heat equation by a theorem of Petrovski [8].

The proofs of existence and uniqueness of kernel functions at points satisfying either condition are similar. We shall concentrate on points satisfying condition L2, pointing out any essential changes which would be required in the proofs for points satisfying $\mathrm{L} 1$.

We begin by establishing the following notation: if $(Y, S)$ is a point satisfying condition $\mathrm{L} 2$ with a mixed-Lipschitz constant $C$, we fix a constant $d<2 C$ and define, for sufficiently small $r$,

$$
\Psi((Y, S), r)=D \cap\left\{(x, t):\left|x^{\prime}-Y^{\prime}\right|<r,|t-S|<r^{2},\left|x_{n}-Y_{n}\right|<r d\right\},
$$

with $\Delta((Y, S), r)=\partial D \cap\left\{(x, t):\left|x^{\prime}-Y^{\prime}\right|<r,|t-S|<r^{2}\right\}$, and $A((Y, S), r)$ $=\left(Y^{\prime}, Y_{n}+r d, S+(1+\mu) r^{2}\right)$, where $\mu$ is small and depends only on $D$. $(\mu$ is chosen so that $A((Y, S), r) \in D$ for small $r$.) In the case of a point satisfying condition $\mathrm{L} 1$, we have

$$
\Psi((Y, S), r)=D \cap\left\{(x, t):|x-Y|<r,|t-S|<r^{2}\right\},
$$

with $\Delta((Y, S), r)=\partial D \cap\{(x, t):|x-Y|<r\}$ and $A((Y, S), r)=\left(Y, S+(1+\mu) r^{2}\right)$.

Lemma 1.1. Suppose that $D$ is a regular domain for the heat equation which satisfies condition $\mathrm{L} 2$ at $(Y, S) \in \partial_{p} D$. If $\gamma \in(0,1)$, then there is a constant $C$, depending only on $\gamma$ and the mixed-Lipschitz constant, such that $\omega^{(x, t)}(\Delta((Y, S), r))$ $\geqq C$ for $(x, t) \in \Psi((Y, S), \gamma r)$ as long as $r$ is sufficiently small.

Proof. Let $G=G((Y, S), r)=\left\{(x, t):|t-S|<r^{2},\left|x^{\prime}-Y^{\prime}\right|<r,\left|x_{n}-Y_{n}\right|<r d\right\}$ and $h(x, t)=\omega_{G}^{(x, t)}\left(\left\{(x, t):\left|x^{\prime}-Y^{\prime}\right|<r,|t-S|<r^{2}, x_{n}=Y_{n}-r d\right\}\right)$. For small $r, G \cap D$ 
$=\Psi((Y, S), r)$. By the maximum principle in that set, $\omega_{D}^{(x, t)}(\Delta((Y, S), r)) \geqq h(x, t)$ for $(x, t) \in \Psi((Y, S), r)$. Since $\Psi((Y, S), \gamma r) \subset G((Y, S), \gamma r)$ and

$$
C=\inf _{(x, t) \in G((Y, S), \gamma r)} h(x, t)>0,
$$

we have

$$
\omega_{D}^{(x, t)}(\Delta((Y, S), r)) \geqq C>0 \quad \text { for }(x, t) \in \Psi((Y, S), \gamma r) \quad \text { Q.E.D. }
$$

LEMMA 1.2. Suppose that $D$ is a regular domain for the heat equation which satisfies condition $\mathrm{L} 2$ at $(Y, S) \in \partial_{p} D$. Then there is a constant $C>0$, depending only on the mixed-Lipschitz constant, such that, for $r^{\prime} \in(0, r)$, we have

$$
\omega^{(x, t)}\left(\Delta\left((Y, S), r^{\prime}\right)\right) \leqq C \omega^{A((Y, S), r)}\left(\Delta\left((Y, S), r^{\prime}\right)\right)
$$

for $(x, t) \in D-\Psi((Y, S), r)$ if $r$ is sufficiently small.

Proof. (For convenience, let $\Delta=\Delta((Y, S), r)$ and $\Delta^{\prime}=\Delta\left((Y, S), r^{\prime}\right)$.) Since $\omega^{(x, t)}\left(\Delta^{\prime}\right)=0$ for $(x, t) \in \partial_{p} D-\Delta$, it suffices, by the maximum principle, to prove $\left(^{*}\right)$ for $(x, t) \in D \cap \partial \Psi((Y, S), r)$.

We define sets $\Psi_{k}=\Psi\left((Y, S), 2^{k-1} r^{\prime}\right)$, with $\Delta_{k}=\Delta\left((Y, S), 2^{k-1} r^{\prime}\right)$ and $A_{k}$ $=A\left((Y, S), 2^{k-1} r^{\prime}\right)$ for $k=1,2, \ldots, L$, where $2^{L-1} r^{\prime}<3 r / 4<2^{L} r^{\prime}$. By Harnack's inequality there is a positive constant $C_{1}$, independent of $k$, such that

$$
\omega^{A_{k}}\left(\Delta^{\prime}\right) \leqq C_{1} \omega^{A_{k+1}}\left(\Delta^{\prime}\right) \text { for } k=1,2, \ldots, L .
$$

The main part of the proof will be a demonstration of statements

$$
\mathrm{S}_{k}: \quad \omega^{(x, t)}\left(\Delta^{\prime}\right) \leqq C \omega^{A_{k}}\left(\Delta^{\prime}\right) \quad \text { for }(x, t) \in D-\Psi_{k}
$$

for $k=1,2, \ldots, L-1$. Once this has been done, the conclusion of the lemma will follow from statement $S_{L-1}$ and Harnack's inequality.

If $B_{1}=\left(Y^{\prime}, Y_{n}+r d, S\right)$, Lemma 1.1 (with $\gamma=\frac{1}{2}$ and the parameter $2 d$ in place of d) establishes the existence of a positive constant $C_{2}^{\prime}$ such that $\omega^{B_{1}}\left(\Delta^{\prime}\right) \geqq C_{2}^{\prime}$ if $r$ is sufficiently small. There is another constant $C_{2}^{\prime \prime}>0$ such that $\omega^{A_{1}}\left(\Delta^{\prime}\right) \geqq C_{2}^{\prime \prime} \omega^{B_{1}}\left(\Delta^{\prime}\right)$, and, setting $C_{2}=C_{2}^{\prime} \cdot C_{2}^{\prime \prime}, \omega^{A_{1}}\left(\Delta^{\prime}\right) \geqq C_{2}$. It follows that $\omega^{(x, t)}\left(\Delta^{\prime}\right) \leqq 1 \leqq\left(1 / C_{2}\right) \omega^{A_{1}}\left(\Delta^{\prime}\right)$ for $(x, t) \in D$ and, in particular, for $(x, t) \in D-\Psi_{1}$. This establishes statement $\mathrm{S}_{1}$.

Next, for each point $(y, s)$ in the ring $\left(\partial_{p} \Psi_{2} \cap \partial_{p} D\right)-\Delta_{2}$, we construct an auxiliary function as follows: let

$$
\begin{aligned}
D(y, s)= & \left\{(x, t): x_{n}-Y_{n}>d_{0} r^{\prime} \text { or }|t-S|>r^{\prime 2} \text { or }\left|x^{\prime}-Y^{\prime}\right|>r^{\prime}\right\} \\
& \cap\left\{(x, t): x_{n}-y_{n}>-M\left(|t-s|^{1 / 2}+\left|x^{\prime}-y^{\prime}\right|\right)\right\},
\end{aligned}
$$

where $d_{0}$ and $M$ are positive constants to be chosen in due course. Let $D_{N}(y, s)$ $=D(y, s) \cap\{(x, t):|x-Y|<N,|t-S|<N\}$, and take $h_{N}(x, t)$ to be the caloric measure in $D_{N}(y, s)$ of that part of $\partial_{p} D_{N}(y, s)$ lying on the boundary of the removed rectangle, $\left\{(x, t): x_{n}-Y_{n} \leqq d_{0} r^{\prime},|t-S| \leqq r^{\prime 2},\left|x^{\prime}-Y^{\prime}\right| \leqq r^{\prime}\right\}$. By the maximum principle, $h_{N}$ increases in $D(y, s)$ as $N$ increases. Since $h_{N} \leqq 1$ for each $N$, 
there exists a temperature in $D(y, s), h(x, t)=\operatorname{limit}_{N \rightarrow \infty} h_{N}(x, t)$. Because the $h_{N}$ 's are uniformly bounded and vanish on a common boundary neighborhood of $(y, s)$, a regular point, we have $\operatorname{limit}_{(x, t) \rightarrow(y, s) ;(x, t) \in D(y, s)} h(x, t)=0$. We claim that, for each $(y, s) \in\left(\partial_{p} \Psi_{2} \cap \partial_{p} D\right)-\Delta_{2}$, the rate at which the corresponding $h(x, t)$ tends to zero at $(y, s)$ is the same. To see this, construct

$$
\begin{array}{r}
\bar{D}(y, s)=\left\{(x, t): x_{n}-y_{n}>-M\left(|t-s|^{1 / 2}+\left|x^{\prime}-y^{\prime}\right|\right),\right. \\
\left.\left|x^{\prime}-y^{\prime}\right|<r^{\prime},|t-s|<r^{\prime 2}\right\},
\end{array}
$$

and let $g(x, t)$ be the caloric measure in $\bar{D}(y, s)$ of that part of the boundary of $\bar{D}(y, s)$ not on the parabolic surface. Clearly, $\bar{D}(y, s) \subset D(y, s)$ and, by the maximum principle in $\bar{D}(y, s), h(x, t) \leqq g(x, t)$. Since the regions $\bar{D}(y, s)$ are identical, except for a translation, we have the desired uniform estimate on the functions $h(x, t)$. Accordingly, choose a positive number $\delta$ such that $h(x, t) \leqq 1 / C_{1}$ whenever $(x, t) \in D(y, s)$ with $|(x, t)-(y, s)|<\delta$. Define the set $\beta_{2}=\left\{(x, t):(x, t) \in \partial \Psi_{2}\right.$ $-\partial D$ and dist $\left.\left((x, t),\left(\partial \Psi_{2} \cap \partial_{p} D\right)-\Delta_{2}\right)<\delta\right\}$, where dist $(P, S)$ is the distance from the point $P$ to the set $S$. Let $\alpha_{2}=\partial \Psi_{2}-\beta_{2}$. By Harnack's inequality, there is a constant $C_{3}>0$ such that

$$
\omega^{(x, t)}\left(\Delta^{\prime}\right)<C_{3} \omega^{A_{2}}\left(\Delta^{\prime}\right) \text { for }(x, t) \in \alpha_{2} .
$$

If $C_{4}=\max \left(C_{3}, 1 / C_{2}\right)$, we have already seen that $\omega^{(x, t)}\left(\Delta^{\prime}\right) \leqq C_{4} \omega^{A_{1}}\left(\Delta^{\prime}\right)$ for $(x, t)$ $\in D-\Psi_{1}$. Furthermore, we now see that $\omega^{(x, t)}\left(\Delta^{\prime}\right) \leqq C_{4} \omega^{A_{2}}\left(\Delta^{\prime}\right)$ for $(x, t) \in \alpha_{2}$, by (b). Therefore, to prove $\omega^{(x, t)}\left(\Delta^{\prime}\right) \leqq C_{4} \omega^{A_{2}}\left(\Delta^{\prime}\right)$ in all of $D-\Psi_{2}$, it suffices, by the maximum principle, to prove this estimate for $(x, t) \in \beta_{2}$. Provided that the constants $M$ and $d_{0}$ have been chosen sufficiently large that $\Psi_{1}$ is contained in the complement of $D_{1}(y, s)$ and the parabolic cone in the complement of $D(y, s)$ lies in the complement of $D$, which is made possible by the Lipschitz nature of $\partial_{p} D$, we may proceed. Since $\omega^{(x, t)}\left(\Delta^{\prime}\right) \leqq C_{4} \omega^{A_{1}}\left(\Delta^{\prime}\right)$ for $(x, t) \in D \cap \partial D_{1}(y, s)$, the maximum principle in $D \cap D_{1}(y, s)$ implies that $\omega^{(x, t)}\left(\Delta^{\prime}\right) \leqq C_{4} \omega^{A_{1}}\left(\Delta^{\prime}\right) h_{1}(x, t)$ in that set. Recalling (a) and our previous estimate on $h(x, t)$ for $(x, t) \in \beta_{2}$, we have $\omega^{(x, t)}\left(\Delta^{\prime}\right) \leqq C_{4} \omega^{A_{2}}\left(\Delta^{\prime}\right)$ for $(x, t) \in \beta_{2}$ and, therefore, in $D-\Psi_{2}$, establishing statement $S_{2}$. Continuing inductively, the statements $S_{3}, S_{4}, \ldots, S_{L}$ are established, each with the same constant $C=C_{4}$. Q.E.D.

The next lemma is of fundamental importance.

LEMma 1.3. Suppose that $D_{T}$ is a regular domain for the heat equation which satisfies condition $\mathrm{L} 2$ at $(Y, S) \in \partial_{p} D_{T}$. Then there is a positive constant $C$, depending only on the mixed-Lipschitz constant, such that for each neighborhood $N$ of $(Y, S)$ in $\Psi((Y, S), r / 4)$ for which $D_{T}-N$ is regular and points of $N$ also satisfy condition L2 with the same mixed-Lipschitz constant as $\partial_{p} D_{T}$ at $(Y, S)$, if $u(x, t)$ is a nonnegative temperature in $D_{T}-N$ which is continuous in the closure of that set and vanishes on $\partial_{p} D_{T}-\bar{N}$, then

$$
u(x, t) \leqq C u(A((Y, S), r)) \omega^{(x, t)}(\Delta((Y, S), r))
$$

for $(x, t) \in D_{T}-\Psi\left((Y, S),(1+\mu)^{1 / 2} r / 4\right)$ if $r$ is sufficiently small, depending on $(Y, S)$. 
Proof. Fix $\lambda \in(0,1)$ and let $\Delta^{\prime}=\Delta\left((\bar{Y}, \bar{S}), r^{\prime}\right)$ for $(\bar{Y}, \bar{S}) \in \Delta((Y, S), r)$ and $r^{\prime}<\lambda r$. By Lemma 1.2, if $r$ is small,

$$
\omega^{(x, t)}\left(\Delta^{\prime}\right) \leqq C \omega^{A} \lambda\left(\Delta^{\prime}\right) \quad \text { for }(x, t) \in D-\Psi((\bar{Y}, \bar{S}), \lambda r)
$$

where $A_{\lambda}=\left(\bar{Y}^{\prime}, \bar{Y}_{n}+r d, \bar{S}+\lambda^{2} r^{2}(1+\mu)\right)$. In particular,

$$
\omega^{(x, t)}\left(\Delta^{\prime}\right) \leqq C \omega^{A_{\lambda}}\left(\Delta^{\prime}\right) \quad \text { for }(x, t) \in D-\Psi((Y, S),(1+\lambda) r) .
$$

If $\lambda$ is small, $\bar{S}+\lambda^{2} r^{2}(1+\mu) \leqq S+r^{2}+\lambda^{2} r^{2}(1+\mu)<S+(1+\mu / 2) r^{2}$, and Harnack's inequality applies to show that

$$
\omega^{A_{\lambda}}\left(\Delta^{\prime}\right) \leqq C \omega^{A}\left(\Delta^{\prime}\right), \quad \text { where } A=A((Y, S), r) .
$$

If we also require that $\lambda<(1+\mu)^{1 / 2}-1$, we have

$$
\omega^{(x, t)}\left(\Delta^{\prime}\right) \leqq C \omega^{A}\left(\Delta^{\prime}\right) \text { for }(x, t) \in D-\Psi\left((Y, S),(1+\mu)^{1 / 2} r\right) .
$$

Next, let $(\bar{x}, \bar{t}) \in D_{T}-\Psi\left((Y, S),(1+\mu)^{1 / 2} r\right)$. By Besicovitch's results on the differentiation of measures [1],

$$
\frac{d \omega^{(z, t)}}{d \omega^{(X, T)}}(\bar{Y}, \bar{S})=\operatorname{limit}_{\Delta^{\prime} \rightarrow((Y, S)\}} \frac{\omega^{(x, t)}\left(\Delta^{\prime}\right)}{\omega^{(X, T)}\left(\Delta^{\prime}\right)}
$$

for a.e. $\left(\omega^{(X, T)}\right)(\bar{Y}, \bar{S}) \in \Delta((Y, S), r)$. Therefore,

$$
\left(d \omega^{(x, t)} / d \omega^{(X, T)}\right)(\bar{Y}, \bar{S}) \leqq C\left(d \omega^{A} / d \omega^{(X, T)}\right)(\bar{Y}, \bar{S})
$$

for a.e. $\left(\omega^{(X, T)}\right)(\bar{Y}, \bar{S}) \in \Delta((Y, S), r)$, where $C$ is independent of $(\bar{Y}, \bar{S}) \in \Delta((Y, S), r)$.

If $u(x, t)$ is a nonnegative temperature in $D_{T}$, continuous in $\bar{D}_{T}$, and vanishing on $\partial_{p} D_{T}-\Delta((Y, S), r)$, we have

$$
u(\bar{x}, \bar{t})=\int_{\Delta((Y, S), r)} u(\bar{Y}, \bar{S}) d \omega^{(x, t)}(\bar{Y}, \bar{S}) .
$$

Since $d \omega^{(x, t)}=\left(d \omega^{(x, t)} / d \omega^{(X, T)}\right) d \omega^{(X, T)}$, it follows that

$$
u(\bar{x}, \bar{t}) \leqq C u(A) \quad \text { for }(\bar{x}, \bar{t}) \in D_{T}-\Psi\left((Y, S),(1+\mu)^{1 / 2} r\right) .
$$

Applying this argument to the function $u(x, t)$ given in the hypotheses and the region $D_{T}-N$, with $r$ replaced by $r / 4$, and making use of Lemma 1.1 and Harnack's inequality, we obtain

$$
\begin{aligned}
& u(x, t) \leqq C u(A((Y, S), r)) \omega^{(x, t)}(\Delta((Y, S), r)) \\
& \quad \text { for }(x, t) \in D_{T}-\Psi\left((Y, S),(1+\mu)^{1 / 2} r / 4\right) . \quad \text { Q.E.D. }
\end{aligned}
$$

We will most often use the following form of Lemma 1.3:

LEMMA 1.4. Suppose that $D$ is a regular domain for the heat equation which satisfies condition $\mathrm{L} 2$ at $(Y, S) \in \partial_{p} D_{T}$. Then there is a positive constant $C$, depending only on $D$ and the mixed-Lipschitz constant at $(Y, S)$, such that, if $u(x, t)$ is a nonnegative temperature in $D$ which vanishes on $\partial_{p} D-\Delta((Y, S), r / 8)$ for sufficiently small $r$, we then have $u(x, t) \leqq C u(A((Y, S), r)) \omega^{(x, t)}(\Delta((Y, S), r))$ for $(x, t)$ $\in D-\Psi\left((Y, S),(1+\mu)^{1 / 2} r / 4\right)$. 
Proof. Let $r_{0}$ satisfy the requirements of Lemma 1.3. For $r<r_{0}$, let $N$ be a neighborhood of $(Y, S)$ in $\Psi((Y, S), r / 4)$ such that $\Delta((Y, S), r / 8) \subset \partial N \cap \partial D$ and $\partial_{p}\left(D_{T}-N\right)$ satisfies condition L2 with a mixed-Lipschitz constant at points of $N$ which is no bigger than the mixed-Lipschitz constant for $D$ at $(Y, S)$. Application of Lemma 1.3 and the maximum principle yield the desired result. Q.E.D.

We can now prove the existence of kernel functions for a domain $D$ if each point $(Y, S) \in \partial_{p} D$ satisfies condition L1 or conditon L2. Let $(X, T)$ be the normalization point for the kernel functions with $T>S$ and consider a sequence of positive numbers $r_{n}$ which decrease to zero. Let $\Delta_{n}=\Delta\left((Y, S), r_{n}\right)$ and set

$$
v_{n}(x, t)=\omega^{(x, t)}\left(\Delta_{n}\right) / \omega^{(X, T)}\left(\Delta_{n}\right) .
$$

Each $v_{n}$ is a nonnegative temperature in $D$ and $v_{n}(y, s)=0$ for $(y, s) \in \partial_{p} D-\Delta_{n}$. If $r$ is small enough for Lemma 1.4 to hold, there is a number $n_{0}$ such that $v_{n}$ satisfies the hypotheses of that lemma for $n>n_{0}$. Therefore, if $A=A((Y, S), r)$ and $\Delta=\Delta((Y, S), r)$, we have

$$
v_{n}(x, t) \leqq C v_{n}(A) \omega^{(x, t)}(\Delta) \text { for }(x, t) \in D-\Psi\left((Y, S),(1+\mu)^{1 / 2} r / 4\right) .
$$

By Harnack's inequality, $v_{n}(A) \leqq C v_{n}(X, T)=C$. Furthermore, since $\omega^{(x, t)}(\Delta) \leqq 1$, we have $v_{n}(x, t) \leqq C$ for $(x, t) \in D-\Psi_{0}$, where $\Psi_{0}=\Psi\left((Y, S),(1+\mu)^{1 / 2} r / 4\right)$. The Ascoli theorem assures the existence of a subsequence of the functions $v_{n}$ which converge uniformly on compact subsets of $D$. By Harnack's convergence theorem, the limit function $K(x, t)$ must be a temperature in $D$. Clearly, $K(x, t) \geqq 0$ and $K(X, T)=1$. Finally, to see that $K(x, t)$ vanishes on $\partial_{p} D-\{(Y, S)\}$, we need only let $n$ tend to infinity in $\left(^{*}\right)$. Since $r$ may be chosen arbitrarily small, it follows that $K(x, t)$ is a kernel function for the heat equation in $D$ at $(Y, S) \in \partial_{p} D$.

At this point we remark that Lemmas 1.1 through 1.4 can be similarly proven for points $(Y, S) \in \partial_{p} D$ which satisfy condition $\mathrm{L} 1$. The specific changes required are that

$$
\begin{aligned}
D(y, s)= & \left\{(x, t):|x-Y|>r^{\prime} \text { or } t>d_{0} r^{\prime 1 / 2}\right\} \\
& \cap\left\{(x, t): t>-M|x-y|^{2}\right\}
\end{aligned}
$$

in Lemma 1.2 and that $A=\left(\bar{Y}, S+\lambda^{2} r^{2}(1+\mu)\right)$ in Lemma 1.3.

Our next goal is to establish the uniqueness of kernel functions. Before proceeding with the actual proof, however, we will be able to simplify the form of the domain with which we must deal. Again, we consider kernel functions at a point $(Y, S) \in$ $\partial_{p} D$ with respect to a fixed point $(X, T) \in D$ with $T>S$. Generally, $D$ is a regular domain for the Dirichlet problem for the heat equation which satisfies condition L1 or condition $\mathrm{L} 2$ at $(Y, S)$.

Lemma 1.5. Suppose that $D^{*} \subset D$ are both domains which are regular for the heat equation with $(Y, S) \in \partial_{p} D^{*} \cap \partial_{p} D$ and $N$ a neighborhood of $(Y, S)$ such that $N \cap \partial_{p} D^{*}=N \cap \partial_{p} D$. If there is at most one kernel function in $D^{*}$ at $(Y, S)$ with respect to $\left(X^{*}, T^{*}\right) \in D^{*}$ with $T^{*}>S$, then there is at most one kernel function in $D$ at $(Y, S)$ with respect to $(X, T) \in D$ with $T>S$. 
Proof. Suppose that $u$ and $v$ are both kernel functions at $(Y, S)$ in $D$. Define $P u$ to be the solution of the Dirichlet problem for the heat equation in $D^{*}$ with boundary values

$$
\begin{aligned}
P u(y, s) & =u(y, s) & & \text { for }(y, s) \in \partial_{p} D^{*} \cap D, \\
& =0 & & \text { for }(y, s) \in \partial_{p} D^{*} \cap \partial_{p} D .
\end{aligned}
$$

By the maximum principle, $P u \leqq u$ in $D^{*}$, so that $u-P u$ is a nonnegative temperature in $D^{*}$ with zero boundary values on $\partial_{p} D^{*}-\{(Y, S)\}$. Consequently, the normalized function $(u(x, t)-P u(x, t)) /\left(u\left(X^{*}, T^{*}\right)-P u\left(X^{*}, T^{*}\right)\right)$ is a kernel function in $D^{*}$ at $(Y, S)$ with respect to $\left(X^{*}, T^{*}\right)$. By the same reasoning, the analogous function with $u$ replaced everywhere by $v$ is also a kernel function in $D^{*}$ at $(Y, S)$ with respect to $\left(X^{*}, T^{*}\right)$. Since the uniqueness of kernel functions in $D^{*}$ is assumed, we must have

$$
u(x, t)-P u(x, t)=C(v(x, t)-P v(x, t)) \text { in } D^{*},
$$

where $C=\left(u\left(X^{*}, T^{*}\right)-P u\left(X^{*}, T^{*}\right)\right) /\left(v\left(X^{*}, T^{*}\right)-P v\left(X^{*}, T^{*}\right)\right)$. It follows that $u-C v=P u-C P v=P(u-C v)$ in $D^{*}$, where $P(u-C v)$ is defined in the obvious manner, vanishing on $\partial_{p} D^{*} \cap \partial_{p} D$. Because it is also true that $u-C v$ vanishes on $\partial_{p} D-\partial_{p} D^{*}$, the maximum principle implies that $u-C v \equiv 0$ in $D$. Evaluation at $(X, T)$ then reveals that $C=1$. Q.E.D.

This result enables us to reduce the question of uniqueness for kernel functions at $(Y, S)$ in $D$, a regular domain for the heat equation which satisfies condition L1 or condition $\mathrm{L} 2$ at $(Y, S)$, to a question of uniqueness for kernel functions at $(Y, S)$ in the domain $D^{*}=\Psi((Y, S), r)$, with arbitrarily small positive $r$. In this case, $\partial_{p} D^{*}-\partial_{p} D$ has a simplified form; in particular, one "end" of $D^{*}$ lies in the hyperplane $x_{n}=Y_{n}+r d$. We shall next show that $D^{*}$ satisfies an additional condition.

Definition. A bounded region $D_{T}$ in $R^{n+1}$ is parabolically starlike at $(X, T)$ if, for each $(y, s) \in \partial_{p} D_{T}$, there exists a finite parabolic ray with vertex $(y, s)$ and endpoint $(X, T)$ which is contained in $D_{T}$. (We allow the degenerate case in which the parabolic ray becomes a vertical line segment.)

We also require the notion of a parabolic cone.

DEFINITION. $\Gamma$ is a parabolic cone with vertex $(y, s)$ if one of the following holds for some triple of positive constants, $C, C^{\prime}$, and $C^{\prime \prime}$, and for some choice of local space coordinates:

$$
\Gamma=\left\{(x, t): C>x_{n}-y_{n}>C^{\prime}\left|x^{\prime}-y^{\prime}\right|+C^{\prime \prime}|t-s|^{1 / 2}\right\}
$$

or

$$
\Gamma=\left\{(x, t): C>t-s>C^{\prime}|x-y|^{2}\right\} .
$$

It is clear that, for each point $(y, s) \in \partial_{p} D_{T}$ satisfying condition L1 or condition L2, there exists a parabolic cone $\Gamma$ with vertex $(y, s)$ which lies in $D_{T}$. Furthermore, 
if $(Y, S)$ is such a point, the intersection of $\Psi((Y, S), r)$ with the union of parabolic cones with vertices in $\Delta((Y, S), r)$ is a parabolically starlike region. As we have seen, it suffices to prove the uniqueness of kernel functions at $(Y, S)$ in such a region.

Lemma 1.6. Suppose that conditon $\mathrm{L} 2$ is satisfied at $(Y, S) \in \partial_{p} D$ and that $\Psi=\Psi\left((Y, S), r_{0}\right)$ is parabolically starlike at $(X, T)$. Then there is a positive constant $C$ such that, if $u(x, t)$ is any kernel function at $(Y, S)$ in $\Psi$ with respect to $(X, T)$, we have

$$
u(x, t) \geqq C K(x, t) \quad \text { for }(x, t) \in \Psi,
$$

where $K(x, t)$ is the kernel function at $(Y, S)$ in $\Psi$ with respect to $(X, T)$ given by $\operatorname{limit}_{n \rightarrow \infty} \omega^{(x, t)}\left(\Delta_{n}\right) / \omega^{(X, T)}\left(\Delta_{n}\right)$, with $\omega$ denoting caloric measure in $\Psi$ and $\Delta_{n}$ $=\Delta((Y, S), 1 / n)$.

Proof. Since the result is trivially true for $t<S$, we will assume that $S=0$, so that $(Y, S)$ is a point on the "bottom edge" of $D$. For convenience, we will also assume that $Y=0$, and, by a suitable choice of space coordinates, that the parabolic arc from $(0,0)$ to $(X, T)$ is defined by $x_{n}=\gamma t^{1 / 2}$, with $x^{\prime}=0$, for some positive constant $\gamma$.

For $r \in\left(0, r_{0}\right)$, define $\beta=\beta(r)=1-(3(1+\mu))^{1 / 2} \gamma^{-1 / 2} r$, and set $u_{r}(x, t)=u\left(Q_{r}(x, t)\right)$ $=u\left(\beta x^{\prime}, \beta x_{n}+(1-\beta)+B r^{1 / 2}, \beta^{2} t+\gamma(1-\beta)^{2}\right)$, with $B$ to be chosen. Note that $u_{r}$ is a temperature in $Q_{r}^{-1}(D)$. Next, define $\Psi^{r}=\Psi \cap\left\{(x, t):\left(x^{\prime}, x_{n}+(1-\beta)+B r^{1 / 2}, t\right)\right.$ $\in \Psi\}$. For small $r$, we claim that $Q_{r}\left(\Psi^{r}\right) \subset \Psi$, which is evident away from $(Y, S)$. Thus, it suffices to show that $Q_{r}\left(\Psi^{r}\right) \subset\left\{(x, t): x_{n}>f\left(x^{\prime}, t\right)\right\}$, where $f\left(x^{\prime}, t\right)$ is the mixed-Lipschitz function defining the boundary of $D$ near $(Y, S)$. Specifically, we are requiring that

$$
\beta f\left(y^{\prime}, s\right)+(1-\beta)+B r^{1 / 2}>f\left(\beta y^{\prime}, \beta^{2} s+\gamma(1-\beta)^{2}\right)
$$

for $\left(y^{\prime}, s\right)$ near $(0,0)$. It is already known that there is a positive constant $C$ such that

$$
f\left(y^{\prime}, s\right)-f\left(\beta y^{\prime}, \beta^{2} s+\gamma(1-\beta)^{2}\right) \geqq-C\left((1-\beta)\left|y^{\prime}\right|+\left(\left(1-\beta^{2}\right) s+\gamma(1+\beta)^{2}\right)^{1 / 2}\right) .
$$

Consequently, it is enough to show that

$$
(\beta-1) f\left(y^{\prime}, s\right)+(1-\beta)+B r^{1 / 2}-C\left((1-\beta)\left|y^{\prime}\right|+\left(\left(1-\beta^{2}\right) s+\gamma(1-\beta)^{2}\right)^{1 / 2}\right)>0 .
$$

For small $r$, if we make use of the definition of $\beta$,

$$
C\left((1-\beta)\left|y^{\prime}\right|+\left(\left(1-\beta^{2}\right) s+(1-\beta)^{2} \gamma\right)^{1 / 2}\right) \leqq C_{1} r^{1 / 2}
$$

and

$$
(1-\beta) f\left(y^{\prime}, s\right) \leqq(3(1+\mu) / \gamma)^{1 / 2} r^{1 / 2} \cdot C(T+L),
$$

where $C$ is here the mixed-Lipschitz constant at $(Y, S)$ and $L$ is the (space) diameter of $\Psi$. Thus, if $B$ is chosen sufficiently large, we do have $Q_{r}\left(\Psi^{r}\right) \subset \Psi$, and it 
follows that $u_{r}$ is a temperature in $\Psi^{r}$. Furthermore, $u_{r}$ is continuous in the closure of $\Psi^{r}$, so that

$$
u_{r}(x, t)=\int_{\partial_{p} \Psi r} u_{r}(y, s) d \omega_{r}^{(x, t)}(y, s) \quad \text { for }(x, t) \in \Psi^{r},
$$

where $\omega_{r}$ denotes caloric measure in $\Psi^{r}$. It follows that

$$
u_{r}(x, t) \geqq \inf _{(y, s) \in \Delta_{r}} u_{r}(y, s) \cdot \omega_{r}^{(x, t)}\left(\Delta_{r}\right) \quad \text { for }(x, t) \in \Psi^{r r},
$$

where $\Delta_{r}=\Delta((Y, S), r)$.

For $(y, s) \in \Delta_{r}, Q_{r}(y, s)$ has $t$-coordinate

$$
\beta^{2} s+\gamma(1-\beta)^{2} \geqq-\beta^{2} r^{2}+3(1+\mu) r^{2} \geqq(2+3 \mu) r^{2} .
$$

Recalling that the point $A_{r}=A((Y, S), r)$ has $t$-coordinate $(1+\mu) r^{2}$, we see that Harnack's inequality will imply $\inf _{(y, s) \in \Delta_{r}} u_{r}(y, s) \geqq C u\left(A_{r}\right)$, with a constant $C$ independent of $r$, provided only that $Q_{r}(y, s)$ is bounded away from $\partial \Psi$ in the space variables by a fixed multiple of $r$ for points $(y, s) \in \Delta_{r}$. Since $Q_{r}(y, s)$ $=Q_{r}\left(y^{\prime}, f\left(y^{\prime}, s\right), s\right)=\left(\beta y^{\prime}, \beta f\left(y^{\prime}, s\right)+(1-\beta)+B r^{1 / 2}, \beta^{2} s+\gamma(1-\beta)^{2}\right)$, this requirement is satisfied by our choice of $B$ above. We then have

$$
u_{r}(x, t) \geqq C u\left(A_{r}\right) \omega_{r}^{(x, t)}\left(\Delta_{r}\right) \quad \text { for }(x, t) \in \Psi^{r} .
$$

By Lemma 1.4, there is a constant $C_{0}>0$ such that

$$
1=u(X, T) \leqq C_{0} u\left(A_{r}\right) \omega^{(x, t)}\left(\Delta_{r}\right),
$$

$\omega$ denoting caloric measure in $\Psi$. Thus,

$$
u_{r}(x, t) \geqq C \omega_{r}^{(x, t)}\left(\Delta_{r}\right) / \omega^{(X, T)}\left(\Delta_{r}\right) \quad \text { for }(x, t) \in \Psi^{r} .
$$

We allow $r$ to range through the sequence $1 / n, n=1,2, \ldots$ According to the maximum principle,

$$
\omega_{n}^{(x, t)}\left(\Delta_{n}\right) \geqq \omega^{(x, t)}\left(\Delta_{n}\right)-\sup _{(z, u) \in \partial \Psi^{n} \cap \Psi} \omega^{(z, u)}\left(\Delta_{n}\right) \quad \text { for }(x, t) \in \Psi^{n},
$$

where the " $n$ " notation refers in each case to $r=1 / n$. Combining this with our estimate for $u_{r}$ above, we have

$$
u_{n}(x, t) \geqq C \omega^{(x, t)}\left(\Delta_{n}\right) / \omega^{(X, T)}\left(\Delta_{n}\right)-\sup _{(z, u) \in \partial \Psi^{n} \cap \Psi} \omega^{(z, u)}\left(\Delta_{n}\right) / \omega^{(X, T)}\left(\Delta_{n}\right)
$$

for $(x, t) \in \Psi^{n}$. As $n$ tends to infinity, $\Psi^{n}$ tends to $\Psi$ and $u_{n}$ tends to $u$. Thus, to conclude that $u \geqq C K$ in $\Psi$, we need only show that

$$
\sup _{(z, u) \in \partial \Psi^{n} \cap \Psi} \omega^{(z, u)}\left(\Delta_{n}\right) / \omega^{(X, T)}\left(\Delta_{n}\right)
$$

tends to zero as $n$ tends to infinity. This follows from Lemma 1.4 and another application of Harnack's inequality. Q.E.D. 
THEOREM 1.7. Suppose that $D$ is a regular domain for the heat equation which satisfies condition $\mathrm{L} 2$ at $(Y, S) \in \partial_{p} D$. If $(X, T) \in D$ with $T>S$, then there is a unique kernel function at $(Y, S)$ in $D$ with respect to $(X, T)$.

Proof. We have previously established the existence of a kernel function at $(Y, S)$ in $D$ with respect to $(X, T)$ in the form of a limit of normalized caloric measures. We will continue to denote this kernel function by $K(x, t)$. Our present task is then reduced to a proof of the uniqueness of $K(x, t)$. According to Lemma 1.5, we may assume that $D$ is a set of the form $\Psi((Y, S), r)$. Then, by Lemma 1.6, if $u(x, t)$ is any kernel function at $(Y, S)$ in $D$ with respect to $(X, T)$, then $u(x, t)$ $\geqq C K(x, t)$ for $(x, t) \in D$, where $C$ is a constant independent of $u(x, t)$.

Let

$C_{0}=\sup \{C: u(x, t) \geqq C K(x, t)$ for $(x, t) \in D$,

for every kernel function $u(x, t)$ at $(Y, S)$ in $D$ with respect to $(X, T)\}$.

Clearly, $u \geqq C_{0} K$ for every kernel function $u$ at $(Y, S)$ in $D$ with respect to $(X, T)$. Furthermore, $C_{0} \leqq 1$. If $C_{0}=1$, the strong maximum principle implies that $u=K$. Assuming $C_{0}<1, u_{0}=\left(u-C_{0} K\right) /\left(1-C_{0}\right)$ is another kernel function at $(Y, S)$ in $D$ with respect to $(X, T)$, in which case $u_{0} \geqq C_{0} K$ in $D$. This leads to a new inequality for $u, u \geqq\left(2 C_{0}-C_{0}^{2}\right) K$ in $D$. However, $2 C_{0}-C_{0}^{2}>C_{0}$, contradicting our assumption that $C_{0}$ is the maximal constant satisfying $u \geqq C K$ in $D$ for every kernel function $u$ at $(Y, S)$ in $D$ with respect to $(X, T)$. Q.E.D.

REMARK 1.8. We have given proofs of the existence and uniqueness of kernel functions at a point $(Y, S)$ of the parabolic boundary of $D$ only in the case that condition L2 is satisfied at $(Y, S)$. For points satisfying condition L1 ("bottom" points of $D$ ), existence of a kernel function is established in corresponding manner. In this case, the uniqueness of the kernel function follows from the uniqueness of the kernel function at the center base point of a cylinder, which can be verified along lines similar to those of Lemma 1.6. Assuming that the point in question is $(0,0)$, and that $u(x, t)$ is an arbitrary kernel function at that point, the approximating functions are defined to be

$$
u_{r}(x, t)=u\left(\beta x, \beta^{2} t+(1-\beta)^{2}\right), \quad \text { with } \beta=1-(3(1+\mu))^{1 / 2} r .
$$

Only the details of the proof differ from the previous one.

REMARK 1.9. As an easy consequence of Theorem 1.7, we see that the kernel function at a point $(Y, S) \in \partial_{p} D$ with respect to $(X, T)$ is equal to the RadonNikodym derivative, $d \omega^{(x, t)} / d \omega^{(X, T)}$, evaluated at $(Y, S)$. Furthermore, if we now emphasize the dependence of the kernel function on the point $(Y, S)$ by denoting the kernel function at $(Y, S)$ in $D$ with respect to $(X, T)$ by $K(x, t, Y, S)$, it is easily seen that this dependence is continuous for $(Y, S)$ on the parabolic boundary.

With the notion of kernel functions well established, we can now prove an important representation theorem for nonnegative temperatures. 
THEOREM 1.10. Suppose that $D$ is a domain which satisfies condition L1 or condition L2 at each point of $\partial_{p} D$. If $u(x, t)$ is a nonnegative temperature in $D_{T}=D$ $\cap\{(x, t): t<T\}$, then there is a unique Borel measure $v$ on $\partial_{p} D_{T}$ such that

$$
u(x, t)=\int_{\partial_{p} D_{T}} K(x, t, y, s) d \nu(y, s)
$$

where $K(x, t, y, s)$ is the kernel function at $(y, s)$ in $D$ with respect to a fixed point $(X, T) \in D$.

Proof. For any relatively closed subset $B$ of $D_{T}$, define, for $(x, t) \in D_{T}, R_{u}^{B}(x, t)$ $=\inf \left\{v(x, t): v\right.$ is a nonnegative supercaloric function in $D_{T}$ with $v \geqq u$ on $\left.B\right\}$. (A supercaloric function is a super-solution of the heat equation.) For $(x, t) \in B$, $R_{u}^{B}(x, t)=u(x, t)$, and, for $(x, t) \in D_{T}-B, R_{u}^{B}(x, t)$ is equal to the Wiener solution of the Dirichlet problem for the heat equation with boundary values $u$ on $\partial B \cap D_{T}$ and zero on the closure of $\partial_{p} D_{T}-B$.

Next, for $(x, t) \in D_{T}$ and $F$ a closed subset of $\partial_{p} D_{T}$, define

$$
\bar{\nu}^{(x, t)}(F)=\inf \left\{R_{u}^{\bar{U} \cap D}(x, t): U \text { is an open subset of } \boldsymbol{R}^{n+1}, F \subset U\right\} .
$$

For any sequence of open sets $U_{i}$ which decrease to $F$, we have

$$
\bar{\nu}^{(x, t)}(F)=\operatorname{limit}_{i \rightarrow \infty} R_{u}^{U_{i} \cap D}(x, t) .
$$

By Harnack's monotone convergence theorem, $\tilde{\nu}^{(x, t)}(F)$ is a nonnegative temperature in $D_{T}$.

For fixed $(x, t) \in D_{T}, \bar{\nu}^{(x, t)}(F)$ is a nonnegative, monotone, subadditive function on the closed subsets of $\partial_{p} D_{T}$ which is additive on disjoint closed sets. Moreover, $\bar{\nu}^{(x, t)}(F)$ is regular:

$$
\bar{\nu}^{(x, t)}(F)=\inf \left\{\bar{\nu}^{(x, t)}(G): G \text { is a closed subset of } \partial_{p} D_{T}, F \subset G^{\circ}\right\} .
$$

(Here, $G^{\circ}$ denotes the interior of $G$.) A standard result implies that $\bar{\nu}^{(x, t)}$ can be extended to a regular Borel measure $\nu^{(x, t)}(\cdot)$ on $\partial_{p} D_{T}$.

By Harnack's inequality, $\nu^{(x, t)}$ is absolutely continuous with respect to $\nu^{(X, T)}$ if $(x, t) \in D_{T}$. Taking $\Delta_{r}=\Delta((y, s), r)$ for $(y, s) \in \partial_{p} D_{T}$, it follows from Lemma 1.4 and the uniqueness of kernel functions that

$$
K(x, t, y, s)=\operatorname{limit}_{r \rightarrow 0} \nu^{(x, t)}\left(\Delta_{r}\right) / \nu^{(X, T)}\left(\Delta_{r}\right)=\frac{d \nu^{(x, t)}}{d \nu^{(X, T)}}(y, s) .
$$

We then have

$$
u(x, t)=\nu^{(x, t)}\left(\partial_{p} D_{T}\right)=\int_{\partial_{p} D_{T}} d \nu^{(x, t)}(y, s)=\int_{\partial_{p} D_{T}} K(x, t, y, s) d \nu^{(X, T)}(y, s) .
$$

To prove uniqueness for the representing measure, let $\eta$ be another regular Borel measure on $\partial_{p} D_{T}$ such that

$$
u(x, t)=\int_{\partial_{p} D_{T}} K(x, t, y, s) d \eta(y, s) .
$$


For a closed subset $F$ of $\partial_{p} D_{T}$, choose a sequence of open sets $G_{k} \subset R^{n+1}$ such that $F=\bigcap G_{k}$ and $\nu^{(X, T)}(F)=\operatorname{limit}_{k \rightarrow \infty} R_{u}^{G_{k} \cap D}(X, T)$. Let $\omega_{k}$ denote the caloric measure in $D-G_{k}$ and let $H_{k}=\partial_{p}\left(D_{T}-\bar{G}_{k}\right)$. For $(x, t) \in D_{T}-\bar{G}_{k}$,

$$
\begin{aligned}
R_{u}^{G_{k} \cap D}(x, t) & =\int_{D \cap H_{k}} u(y, s) d \omega_{k}^{(x, t)}(y, s) \\
& =\int_{D \cap H_{k}}\left(\int_{\partial_{p} D_{T}} K(y, s, z, u) d \eta(z, u)\right) d \omega_{k}^{(x, t)}(y, s) \\
& =\int_{\partial_{p} D_{T}}\left(\int_{D \cap H_{k}} K(y, s, z, u) d \omega_{k}^{(x, t)}(y, s)\right) d \eta(z, u) .
\end{aligned}
$$

For $(z, u) \in F, K(y, s, z, u)$ is a temperature $(y, s)$ in $D_{T}-\bar{G}_{k}$ which is continuous in the closure of that set. For such $(z, u)$,

$$
\int_{D \cap H_{k}} K(y, s, z, u) d \omega_{k}^{(x, t)}(y, s)=K(x, t, z, u) .
$$

For $(z, u) \in \partial_{p} D_{T}-F$,

$$
\operatorname{limit}_{k \rightarrow \infty} \int_{D \cap H_{k}} K(y, s, z, u) d \omega_{k}^{(x, t)}(y, s)=0 .
$$

By the maximum principle,

$$
\int_{D \cap H_{k}} K(y, s, z, u) d \omega_{k}^{(x, t)}(y, s) \leqq K(x, t, z, u) \quad \text { for }(x, t) \in D_{T}-\bar{G}_{k}
$$

and for each value of $k$. Lebesgue's theorem then implies that

$$
v^{(X, T)}(F)=\operatorname{limit}_{k \rightarrow \infty} R_{u}^{G_{k} \cap D}(X, T)=\int_{F} K(X, T, z, u) d \eta(z, u)=\eta(F) .
$$

Since both of the measures are regular, uniqueness is established. Q.E.D.

2. Existence of parabolic limits at the boundary. In this section we will make use of Theorem 1.10 in proving the existence of parabolic limits almost everywhere $\left(\omega^{(X, T)}\right)$ on $\partial_{p} D_{T}$ for nonnegative temperatures in $D_{T}$.

Definition. A function $u(x, t)$ defined in $D_{T}$ has parabolic limit $L$ at a point $(y, s) \in \partial_{p} D_{T}$ if, for each parabolic cone $V \subset D$ with vertex $(y, s)$ which opens away from $\partial D$ and satisfies $\bar{V} \cap \partial_{p} D_{T}=\{(y, s)\}$, we have $\operatorname{limit}_{(x, t) \rightarrow(y, s) ;(x, t) \in V} u(x, t)=L$. (For $(y, s) \in \partial_{p} D_{T}$ satisfying condition L2, $V$ opens away from $\partial D$ if $V \subset\left\{(x, t): x_{n}>y_{n}\right\}$.)

We begin with several lemmas concerning the uniform behavior of kernel functions. We continue to assume condition $\mathrm{L} 1$ or condition $\mathrm{L} 2$ on $\partial_{p} D$.

Lemma 2.1. Let $(Y, S) \in \partial_{p} D$ with $\Delta=\Delta((Y, S), r)$. Then, for sufficiently small $r$,

$$
\sup _{(y, s) \in \partial_{p} D-\Delta} K(x, t, y, s) \rightarrow 0 \quad \text { as }(x, t) \rightarrow(Y, S) \text { in } D .
$$


Proof. (For condition L2 holding at $(Y, S)$.)

Since $K(x, t, y, s) \equiv 0$ for $s \geqq T$, we need only consider points $(y, s)$ in $\partial_{p} D_{T}-\Delta$. For a large positive number $M$, let $\Sigma=\left\{(x, t): x_{n}>-M\left(|t|^{1 / 2}+\left|x^{\prime}\right|\right),|x|<1\right.$, $|t|<1\}$, and let $h(x, t)$ denote the caloric measure in $\Sigma$ of that part of $\partial_{p} \Sigma$ satisfying $|x|=1$ or $|t|=1$. Shrink $\Sigma$ parabolically by the map $(x, t) \rightarrow\left(r x / 2, r^{2} t / 4\right)$ and translate this region in such a way that the origin is moved to the point $(Y, S)$ and the orientation coincides with the local coordinates at $(Y, S)$. If $M$ is large, the cone $\left\{(x, t): x_{n}>-M\left(|t|^{1 / 2}+\left|x^{\prime}\right|\right)\right\}$, after the shrinking and translation, lies in the complement of $D$.

For $s \in(S, T), K(x, t, y, s)=0$ in a neighborhood of $(Y, S)$, so we need only prove the lemma for $(y, s) \in U$, where $U \subset \partial_{p} D_{T}-\Delta$ is bounded away from $\{(x, t): t=T\}$. By Lemma 1.4, if $\left(Y_{0}, S_{0}\right) \in U$ and $\Delta_{0}=\Delta\left(\left(Y_{0}, S_{0}\right), r_{0}\right)$, with $r_{0}<r / 4$, we have

$$
\omega^{(x, t)}\left(\Delta_{0}\right) \leqq C \omega^{A\left(\left(Y_{0}, S_{0}\right), r\right)}\left(\Delta_{0}\right) \text { for }(x, t) \in D-\Psi\left(\left(Y_{0}, S_{0}\right),(1+\mu)^{1 / 2} r / 4\right) .
$$

Furthermore, a careful examination of the proof of Lemma 1.4 reveals that the constant may be chosen to hold for all $\left(Y_{0}, S_{0}\right) \in U$. Applying Harnack's inequality,

$$
\omega^{(x, t)}\left(\Delta_{0}\right) \leqq C \omega^{(X, T)}\left(\Delta_{0}\right) \text { for }(x, t) \in D-\Psi\left(\left(Y_{0}, S_{0}\right),(1+\mu)^{1 / 2} r / 4\right),
$$

with $C$ now depending on $r$ as well as on $D$ and $U$. If $\Sigma_{r}$ denotes the transformed region $\Sigma$, then $D \cap \Sigma_{r} \subset D-\Psi\left(\left(Y_{0}, S_{0}\right),(1+\mu)^{1 / 2} r / 4\right)$. By the maximum principle in $D \cap \Sigma_{r}$,

$$
\omega^{(x, t)}\left(\Delta_{0}\right) \leqq C \omega^{(X, T)}\left(\Delta_{0}\right) h_{r}(x, t) \text { for }(x, t) \in D \cap \Sigma_{r},
$$

where $h_{r}$ is the caloric measure in $\Sigma_{r}$ corresponding to $h$ in $\Sigma$. Since $h_{r}(x, t)$ tends to zero as $(x, t)$ tends to $(Y, S)$, we see that

$$
\omega^{(x, t)}\left(\Delta_{0}\right) / \omega^{(X, T)}\left(\Delta_{0}\right) \rightarrow 0 \quad \text { as }(x, t) \rightarrow(Y, S),
$$

independent of $\left(Y_{0}, S_{0}\right)$ in $U$ and $r_{0}<r / 4$. It follows that

$$
\sup _{(y, s) \in U} K(x, t, y, s) \rightarrow 0 \quad \text { as }(x, t) \rightarrow(Y, S) . \quad \text { Q.E.D. }
$$

In the several lemmas that follow, attention will be restricted to domains $D_{T}$ which satisfy an additional condition:

(Z) There is a hyperplane $H=\{(x, t):\langle x, \alpha\rangle=\theta\}$ such that $\partial_{p} D_{T}$ intersects $H$ in a simply connected set $B$, which is open in $H$, with $\sup _{(x, t) \in B} t=T$ and $\inf _{(x, t) \in B} t=0$.

In particular, $(Z)$ is satisfied by domains $\Psi((Y, S), r)$ with an appropriate choice of local coordinates. In connection with condition $(\mathrm{Z})$, we shall also assume that $\theta=0$ and $D_{T} \subset\{(x, t):\langle x, \alpha\rangle\langle 0\}$. (Here, $\alpha$ is an $n$-vector and $\langle\cdot, \cdot\rangle$ denotes the inner product in $\boldsymbol{R}^{n}$.)

Lemma 2.2. Let $D_{T}$ satisfy $(\mathrm{Z})$ and let $U \subset \partial_{p} D_{T}$ be bounded away from the set $B$. Then

$$
\sup _{\{(x, t, y, s):\langle x, \alpha\rangle>-\varepsilon, t \leqq T,(y, s) \in U\}} K(x, t, y, s) \rightarrow 0 \quad \text { as } \varepsilon \rightarrow 0 .
$$


Proof. Let $D_{\varepsilon_{0}}=D_{T} \cap\left\{(x, t):\langle x, \alpha\rangle<-\varepsilon_{0}\right\}$, where $0<\varepsilon_{0}<\inf _{(y, s) \in U}-\langle y, \alpha\rangle$. By Lemma 2.1 and Harnack's inequality,

$$
\sup _{(z, u) \in D_{T} \cap \partial_{p} D_{\varepsilon_{0}}} K(z, u, y, s) \leqq C K\left(X, T+\varepsilon_{0}, y, s\right) \quad \text { for }(y, s) \in U .
$$

Since $K\left(X, T+\varepsilon_{0}, y, s\right)$ depends continuously on $(y, s)$ in $\partial_{p} D_{T}$, there is a positive constant $M$ such that $\sup _{(y, s) \in U} K\left(X, T+\varepsilon_{0}, y, s\right) \leqq M$. Therefore,

$$
\sup _{(y, s) \in U:(z, u) \in D_{T} \cap \partial_{p} D_{\varepsilon_{0}}} K(z, u, y, s) \leqq C M .
$$

Since $K(z, u, y, s) \rightarrow 0$ as $(z, u) \rightarrow \partial_{p} D_{T}-U$ for $(y, s) \in U$, the maximum principle implies that

$$
K(z, u, y, s) \leqq M(z, u) \text { for }(z, u) \in D_{T}-D_{\varepsilon_{0}},
$$

where $M(z, u)$ is the unique bounded temperature in $D_{T}-D_{\varepsilon_{0}}$ with boundary values equal to $C M$ for $(z, u) \in D_{T} \cap \partial_{p} D_{\varepsilon_{0}}$ and equal to zero for $(z, u) \in \partial_{p} D_{T} \cap \partial_{p} D_{\varepsilon_{0}}$. Clearly,

$$
\sup _{(z, u) \in D_{r}-D_{\varepsilon}} M(z, u) \rightarrow 0 \quad \text { as } \varepsilon \rightarrow 0 \text {. Q.E.D. }
$$

Lemma 2.3. Again let $D_{T}$ satisfy $(Z)$. Let $U_{0} \in \partial_{p} D_{T}$ be bounded away from the set $B$ and let $D_{T, r}=D_{T} \cap\{(x, t):\langle x, \alpha\rangle<-r\}$. Then there is a positive constant $C$ such that, if $r$ is sufficiently small, $\omega_{T, r}^{(X, T)}(U) \geqq C \omega^{(X, T)}(U)$ for each measurable set $U \subset U_{0}$, where the caloric measures are taken in $D_{T, r}$ and $D$, respectively.

Proof. For sufficiently small $r,(X, T) \in D_{T, r}$, and we have

$$
\omega_{T, r}^{(X, T)}(U)=\int_{U} W_{r}(y, s) d \omega^{(X, T)}(y, s),
$$

where $W_{r}(y, s)=1-\int_{D_{T} \cap \partial_{p} D_{T, r}} K(z, u, y, s) d \omega_{T, r}^{(X, T)}(z, u), K$ denoting the kernel functions in $D$. To complete the proof, we will show that $W_{r}(y, s) \geqq C>0$ for small $r$ and for $(y, s) \in U_{0}$. Clearly, $W_{r}(y, s) \geqq 1-\sup _{(z, u) \in D_{r} \cap \partial_{p} D_{T, r}:(y, s) \in U_{0}} K(z, u, y, s)$. By Lemma 2.2, there is a constant $r_{0}>0$ such that

$$
\sup _{(z, u) \in D_{T} \cap \partial_{p} D_{T, r} ;(y, s) \in U_{0}} K(z, u, y, s) \leqq \frac{1}{2} \quad \text { for } r<r_{0} . \quad \text { Q.E.D. }
$$

Lemma 2.4. Suppose that $D_{T}$ is a set of the form $\Psi\left(\left(Y_{0}, S_{0}\right), r_{0}\right)$, where condition $\mathrm{L} 2$ is satisfied at $\left(Y_{0}, S_{0}\right)$ for some larger domain. Then there is a positive constant $C$ such that, if $(Y, S) \in \Delta\left(\left(Y_{0}, S_{0}\right), r_{0} / 2\right)$ and $r$ is sufficiently small, we have

$$
K(A, y, s) \leqq C / \omega^{(X, T)}(\Delta((Y, S), r)) \quad \text { for }(y, s) \in \Delta((Y, S), r),
$$

where $A=A((Y, S), r)$.

Proof. From equation $\left({ }^{*}\right)$ in the proof of Lemma 1.6,

$$
K_{r}(x, t, y, s) \geqq C K(A, y, s) \omega_{r}^{(x, t)}(\Delta((Y, S), r))
$$


for $(x, t) \in D^{r}$ and $(y, s) \in \Delta\left(\left(Y_{0}, S_{0}\right), r_{0} / 2\right)$, where the " $r$ " notation corresponds to that lemma. However, each set $D^{r}$ (Lemma 1.6) is equal to a set $D_{T, r^{\prime}}$ (Lemma 2.3) and $r, r^{\prime}$ tend to zero together. Applying Lemma 2.3 with $(x, t)=(X, T)$, we have

$$
K(A, y, s) \leqq C K_{r}(X, T, y, s) / \omega^{(X, T)}(\Delta((Y, S), r))
$$

for $r<r_{1}$ and $(y, s) \in \Delta\left(\left(Y_{0}, S_{0}\right), r_{0} / 2\right)$, where $r_{1}<r_{0} / 2$. Repeating an argument in the proof of Lemma 2.2, there is a constant $M>0$ such that

$$
\sup _{(y, s) \in \Delta\left(\left(Y_{0}, S_{0}\right), r_{0} / 2\right)} K_{r}(X, T, y, s) \leqq M . \quad \text { Q.E.D. }
$$

Again, a similar result can be proven when condition $\mathrm{L} 1$ is satisfied at a point $(Y, 0) \in \partial_{p} D$. In this case one considers $K_{r}(x, t, y, s)=K\left(x, t+(2+\mu) r^{2}, y, s\right)$. By the maximum principle and Harnack's inequality,

$$
K_{r}(X, T, y, s) \geqq C K(A, y, s) \omega^{(X, T)}(\Delta((Y, 0), r)) \text { for small } r .
$$

We can now prove the final essential lemma.

Lemma 2.5. Again suppose that $D_{T}$ is a set of the form $\Psi\left(\left(Y_{0}, S_{0}\right), r_{0}\right)$, where condition $\mathrm{L} 2$ is satisfied at $\left(Y_{0}, S_{0}\right)$ for some larger domain. Let $V$ be a parabolic cone in $D_{T}$ with vertex $(Y, S) \in \Delta\left(\left(Y_{0}, S_{0}\right), r_{0}\right)$, with $V$ opening away from $\partial D$. Let $(\bar{x}, \bar{t}) \in V$ satisfy $\bar{x}_{n}-Y_{n}=r d$, where $d$ is the fixed parameter in the definition of $\Psi((Y, S), r)$, and let $\Delta_{j}=\Delta\left((Y, S), 2^{j} r\right)$, with $R_{0}=\Delta_{0}$ and $R_{j}=\Delta_{j}-\Delta_{j-1}, j=1,2$, .... For sufficiently small $r$, we then have

$$
\sup _{(y, s) \in R_{j}} K(\bar{x}, \bar{t}, y, s) \leqq C C_{\jmath} / \omega^{(X, T)}\left(\Delta_{j}\right), \quad j=1,2, \ldots, N,
$$

where $N$ is the smallest integer such that $2^{N} r>r_{0}$. The positive constant $C$ depends only on $D_{T}$ and $V$ and the positive constants $C_{j}$ satisfy $\sum_{j=0}^{\infty} C_{j} \leqq C^{\prime}=C^{\prime}\left(D_{T}\right)$.

Proof. Let $A_{j}=A\left((Y, S), 2^{j} r\right)$. By Harnack's inequality, if $u$ is any nonnegative temperature in $D_{T}, u(\bar{x}, \bar{t}) \leqq C u\left(A_{0}\right)$, with the constant depending only on $D_{T}$ and $V$. In particular,

$$
K(\bar{x}, \bar{t}, y, s) \leqq C K\left(A_{0}, y, s\right) \text { for }(y, s) \in \partial_{p} D_{T} .
$$

By Lemma 2.4,

$$
\sup _{(y, s) \in \Delta_{j}} K\left(A_{j}, y, s\right) \leqq C / \omega^{(X, T)}\left(\Delta_{j}\right),
$$

with $C$ independent of $j$. Moreover, for $j=1,2,3,4$, say, Harnack's inequality establishes the existence of constants $C_{j}=C_{j}\left(D_{T}\right)$ such that $K\left(A_{0}, y, s\right)$ $\leqq C_{j} K\left(A_{j}, y, s\right)$. Therefore,

$$
\sup _{(y, s) \in R_{j}} K\left(A_{0}, y, s\right) \leqq C C_{j} / \omega^{(X, T)}\left(\Delta_{j}\right) \quad \text { for } j=1,2,3,4 .
$$


Now, let $\left(y_{j}, s_{j}\right) \in R_{j}$ for $j>4$ and suppose $\Delta \subset \Delta_{j}^{\prime}=\Delta\left(\left(y_{j}, s_{j}\right), r_{j}\right)$, where $r_{j}=2^{j-4} r$. Let $A_{j}^{\prime}=A\left(\left(y_{j}, s_{j}\right), r_{j}\right)$. By Lemma 1.4 ,

$$
K\left(x, t, y_{j}, s_{j}\right) \leqq C K\left(A_{j}^{\prime}, y_{j}, s_{j}\right) \text { for }(x, t) \in D_{T}-\Psi_{j},
$$

where $\Psi_{j}=\Psi\left(\left(y_{j}, s_{j}\right),(1+\mu)^{1 / 2} r_{j} / 4\right)$. Since

$$
\left(2^{j+1} r\right)^{2}(1+\mu)-\left(\left(2^{j} r\right)^{2}+\left(2^{j-4} r\right)^{2}(1+\mu)\right) \geqq r^{2}\left(2^{2 j+2}-2^{2 j+1}\right)=2^{2 j+1} r^{2},
$$

Harnack's inequality can be applied to show $K\left(A_{j}^{\prime}, y_{j}, s_{j}\right) \leqq C K\left(A_{j+1}, y_{j}, s_{j}\right)$. For $(x, t) \in D-\Psi_{j}$, combining this with Lemma 2.4, we have

$$
K\left(x, t, y_{j}, s_{j}\right) \leqq C K\left(A_{j+1}, y_{j}, s_{j}\right) \leqq C / \omega^{(X, T)}\left(\Delta_{j+1}\right) \leqq C / \omega^{(X, T)}\left(\Delta_{j}\right) .
$$

Next, let

$$
\begin{aligned}
\Sigma= & \{(x, t):|x-Y|<1,|t-S|<1\} \\
& \cap\left\{(x, t): x_{n}-Y_{n}>-B\left(|t-S|^{1 / 2}+\left|x^{\prime}-Y^{\prime}\right|\right)\right\},
\end{aligned}
$$

where $B$ is chosen so that $\left\{(x, t): x_{n}-Y_{n} \leqq-B\left(|t-S|^{1 / 2}+\left|x^{\prime}-Y^{\prime}\right|\right)\right\}$ is contained in the complement of $D_{T}$. Let $\Sigma_{j}$ be the region formed by parabolically shrinking $\Sigma$ by the factor $2^{j-3} r$ and let $h_{j}$ denote the caloric measure in $\Sigma_{j}$ of that part of $\partial_{p} \Sigma_{j}$ which does not lie on the cone. The maximum principle in $D_{T} \cap \Sigma_{j}$, which is contained in $D_{T}-\Psi_{j}$, implies that

$$
K\left(x, t, y_{j}, s_{j}\right) \leqq C h_{j}(x, t) / \omega^{(X, T)}\left(\Delta_{j}\right) \quad \text { for }(x, t) \in D_{T} \cap \Sigma_{j} .
$$

Setting $(x, t)=A_{0}, K\left(A_{0}, y_{\jmath}, s_{j}\right) \leqq C h_{j}\left(A_{0}\right) / \omega^{(X, T)}\left(\Delta_{j}\right)$, and it follows that

$$
\sup _{(y, s) \in R,} K(\bar{x}, \bar{t}, y, s) \leqq C h_{j}\left(A_{0}\right) / \omega^{(X, T)}\left(\Delta_{j}\right) .
$$

To complete the proof we must show that $\sum_{j=5}^{\infty} h_{j}\left(A_{0}\right)<\infty$. If $h$ denotes the caloric measure in $\Sigma$ corresponding to $h_{j}$ in $\Sigma_{j}$, we have

$$
\begin{aligned}
h_{j}\left(A_{0}\right) & =h_{j}\left(Y^{\prime}, Y_{n}+r d, S+(1+\mu) r^{2}\right) \\
& =h\left(Y^{\prime}, Y_{n}+2^{3-j} d, S+2^{6-2 j}(1+\mu)\right) .
\end{aligned}
$$

If $m_{0}=\max \left\{h(x, t):(x, t) \in \Sigma,|x-Y| \leqq \frac{1}{2},|t-S| \leqq \frac{1}{4}\right\}$, it is clear that $0<m_{0}<1$. By the maximum principle,

$$
h(Y+(x-Y) / 2, S+(t-S) / 4) \leqq m_{0} h(x, t) \text { for }(x, t) \in \Sigma .
$$

Taking $(x, t)=\left(Y^{\prime}, Y_{n}+2^{3-j} d, S+2^{6-2 j}(1+\mu)\right)$, we have $h_{j+1}\left(A_{0}\right) \leqq m_{0} h_{j}\left(A_{0}\right)$. Thus, by the ratio test, $\sum_{j=5}^{\infty} h_{j}\left(A_{0}\right)<\infty$. Q.E.D.

As usual, the proof for a point $(Y, S)$ at which condition $\mathrm{L} 1$ is satisfied is similar. We omit the details. Using Lemma 2.5, we can now prove the almost everywhere existence of parabolic limits.

THEOREM 2.6. Let $u(x, t)$ be a nonnegative temperature in $D_{T}$, which is assumed to satisfy condition L1 or condition L2 at each point on its parabolic boundary $\partial_{p} D_{T}$. Then $u(x, t)$ has finite parabolic limits at each point $(y, s) \in \partial_{p} D_{T}$, except for a set of zero caloric measure. 
Proof. We shall first prove the theorem in certain simple situations (Cases la and $1 \mathrm{~b}$, below), then combine these for the general result (Case 2, below).

Case 1a. Here $D_{T}$ is assumed to be a set of the form $\Psi\left(\left(Y_{0}, S_{0}\right), r_{0}\right)$. By Theorem 1.10 , there is a unique regular Borel measure $\nu$ on $\delta_{p} D_{T}$ such that

$$
u(x, t)=\int_{\partial_{p} D_{T}} K(x, t, y, s) d v(y, s) .
$$

Furthermore, we have $d \nu(y, s)=f(y, s) d \omega^{(x, T)}(y, s)+d \sigma(y, s)$, where $f(y, s)$ $\in L^{1}\left(\omega^{(X, T)}\right)$ and $\sigma$ is singular with respect to $\omega^{(X, T)}$. In particular, $f(y, s)<\infty$ for $(y, s) \in \partial_{p} D_{T}$ except on a set of zero $\left(\omega^{(X, T)}\right)$ measure. We will show that $u(x, t)$ has parabolic limits equal to $f(y, s)$ almost everywhere $\left(\omega^{(X, T)}\right)$ on $\Delta\left(\left(Y_{0}, S_{0}\right), r_{0}\right)$.

Let $(Y, S) \in \Delta\left(\left(Y_{0}, S_{0}\right), r_{0}\right)$ with $f(Y, S)<\infty$. Then,

$$
\begin{aligned}
u(x, t)-f(Y, S) & =\int_{\partial_{p} D_{T}} K(x, t, y, s)\left(f(y, s) d \omega^{(X, T)}(y, s)+d \sigma(y, s)\right)-f(Y, S) \\
& =\int_{\partial_{p} D_{T}} K(x, t, y, s)\left((f(y, s)-f(Y, S)) d \omega^{(X, T)}(y, s)+d \sigma(y, s)\right) .
\end{aligned}
$$

Let $V$ be a parabolic cone in $D_{T}$ with vertex $(Y, S)$ which opens away from $\partial D$, and let $(x, t) \in V$. Define $\Delta_{j}, R_{j}$, and $A_{j}$ as in Lemma 2.5 for $j=0,1,2, \ldots, N$, where $\Delta_{N-1} \subset \Delta=\Delta\left((Y, S), r_{1}\right) \subset \Delta_{N}$ for some small positive $r_{1}$. Then,

$$
\begin{aligned}
\mid u(x, t)- & f(Y, S) \mid \\
\leqq & \left|\int_{\partial_{p} D_{T}-\Delta} K(x, t, y, s)\left((f(y, s)-f(Y, S)) d \omega^{(X, T)}(y, s)+d \sigma(y, s)\right)\right| \\
& \quad+\sum_{j=0}^{N} \int_{R_{j}} K(x, t, y, s)\left(|f(y, s)-f(Y, S)| d \omega^{(X, T)}(y, s)+d \sigma(y, s)\right) .
\end{aligned}
$$

The second term on the right is dominated by

$$
\sum_{j=0}^{N} \sup _{(y, s) \in R_{j}} K(x, t, y, s)\left(\int_{\Delta_{j}}|f(y, s)-f(Y, S)| d \omega^{(X, T)}(y, s)+\sigma\left(\Delta_{j}\right)\right) .
$$

From Besicovitch's general theory of differentiation of measures,

and

$$
\int_{\Delta_{f}}|f(y, s)-f(Y, S)| d \omega^{(X, T)}(y, s)=o\left(\omega^{(X, T)}\left(\Delta_{f}\right)\right)
$$

$$
\sigma\left(\Delta_{j}\right)=o\left(\omega^{(X, T)}\left(\Delta_{j}\right)\right) \quad \text { as } \Delta_{j} \rightarrow\{(Y, S)\} \quad \text { for a.e }\left(\omega^{(X, T)}\right)(Y, S) \in \Delta\left(\left(Y_{0}, S_{0}\right), r_{0}\right) .
$$

Since $\sup _{(y, s) \in R_{j}} K(x, t, y, s) \leqq C C_{j} / \omega^{(X, T)}\left(\Delta_{j}\right)$ with $\sum_{j=0}^{\infty} C_{j}<\infty$ by the previous lemma, the final term in $\left(^{*}\right)$ can be made small uniformly for $(x, t) \in V$ if $\Delta$ (and, hence, $\left.\Delta_{j}, j=0,1,2, \ldots, N\right)$ is sufficiently small.

Since the first term on the right of $\left({ }^{*}\right)$ can be dominated by

$$
\sup _{(y, s) \in \partial_{p} D_{T}-\Delta} K(x, t, y, s)(\|\nu\|+f(Y, S)),
$$

an application of Lemma 2.1 completes the proof in this case. 
Case 1b. Here $D_{T}=\{(x, t):|x|<1,0<t<1\}$, and $u(x, t)$ is shown to have finite parabolic limits almost everywhere $\left(\omega^{(X, T)}\right)$ on that part of $\partial_{p} D_{T}$ where $t=0$. The proof of Case la can be repeated in this situation, taking into account the remarks made previously concerning boundary points at which condition $\mathrm{Ll}$ is satisfied.

Case 2. In this case we consider general domains $D_{T}$ which satisfy condition $\mathrm{L} 1$ or condition $\mathrm{L} 2$ at each point of $\partial_{p} D_{T}$. Let $\left(y_{i}, s_{i}\right), i=1,2, \ldots$, be a countable dense subset of $\partial_{p} D_{T}$. For each $i$ there is a neighborhood $N_{i}$ of $\left(y_{i}, s_{i}\right)$ in $\partial_{p} D_{T}$ which satisfies one of the following conditions:

(I) If $\left(y_{i}, s_{i}\right)$ is a point of $\partial_{p} D_{T}$ at which condition $\mathrm{L} 1$ is satisfied, then $N_{i}$ is the base of a cylinder $R_{i}$ which is contained in $D_{T}$ and has axis in the $t$-direction.

(II) If $\left(y_{i}, s_{i}\right)$ is a point of $\partial_{p} D_{T}$ at which condition L2 is satisfied, then there is a positive number $r_{i}$ such that $N_{i}=\Delta\left(\left(y_{i}, s_{i}\right), r_{i}\right)$. In this case we take $R_{i}=\Psi\left(\left(y_{i}, s_{i}\right), r_{i}\right)$.

For each $i, N_{i} \subset \partial_{p} R_{i}$, and we can choose another neighborhood $N_{i}^{\prime}$ of $\left(y_{i}, s_{i}\right)$ in $\partial_{p} D_{T}$ with $\bar{N}_{i}^{\prime} \subset N_{i}$. Conditions (I) and (II) guarantee that $u(x, t)$ has finite parabolic limits on $N_{i}^{\prime}$, except possibly for a set of zero caloric measure in $R_{i}$, according to the results of Cases 1a and $1 \mathrm{~b}$. To complete the proof, it suffices to show that if $Z_{i} \subset N_{i}^{\prime}$ is the set of zero caloric measure in $R_{i}$ where $u(x, t)$ fails to have finite parabolic limits, then $Z_{i}$ must also be a set of zero caloric measure in $D_{T}$. With this aim, let $(x, t) \in R_{i}$ with $\omega_{i}^{(x, t)}\left(Z_{i}\right)=0$, where $\omega_{i}$ denotes caloric measure in $R_{i}$. If $\omega$ denotes caloric measure in $D_{T}$, then $\omega^{(x, t)}\left(Z_{i}\right)=P_{i}(x, t)$, where $P_{i}$ is the solution of the Dirichlet problem for the heat equation in $R_{i}$ with boundary values equal to $\omega^{(z, u)}\left(Z_{i}\right)$ for $(z, u) \in \partial_{p} R_{i}-\partial_{p} D_{T}$ and equal to zero for $(z, u) \in \partial_{p} R_{i} \cap \partial_{p} D_{T}$. Since $N_{i} \subset \partial_{p} R_{i} \cap \partial_{p} D_{T}$, we have

$$
\operatorname{limit}_{(x, t) \rightarrow(y, s):(x, t) \in R_{i}} \omega^{(x, t)}\left(Z_{i}\right)=0 \text { for }(y, s) \in N_{i} .
$$

It follows that $\operatorname{limit}_{(x, t) \rightarrow(y, s) ;(x, t) \in D_{T}} \omega^{(x, t)}\left(Z_{i}\right)=0$. Since we also have

$$
\operatorname{limit}_{(x, t) \rightarrow(y, s) ;(x, t) \in D_{T}} \omega^{(x, t)}\left(Z_{i}\right)=0 \text { for }(y, s) \in \partial_{p} D_{T}-N_{i},
$$

the maximum principle implies that $\omega^{(x, t)}\left(Z_{i}\right)=0$ for $(x, t) \in D_{T}$. Q.E.D.

The following result is a simple corollary of the proof.

Corollary 2.7. (I) If $f(y, s) \in L^{1}\left(\partial_{p} D_{T}\right)$ with respect to caloric measure and $f(y, s) \geqq 0$, then the nonnegative temperature

$$
u(x, t)=\int_{\partial_{p} D_{T}} f(y, s) d \omega^{(x, t)}(y, s)
$$

has parabolic limits equal to $f(y, s)$ for almost every $\left(\omega^{(X, T)}\right)(y, s)$ in $\partial_{p} D_{T}$.

(II) $\omega^{(x, t)}(E)$ has parabolic limit equal to 1 almost everywhere $\left(\omega^{(X, T)}\right)$ on $E$, where $E$ is a measurable subset of $\partial_{p} D_{T}$.

REMARKS. Following the arguments of Hunt and Wheeden [4] in the case of harmonic functions, these results can be extended. Specifically, we need only 
require that the temperature $u(x, t)$ have a one-sided bound in some parabolic cone at each point of the parabolic boundary in order that the parabolic limits of $u(x, t)$ exist almost everywhere $\left(\omega^{(X, T)}\right)$ on the parabolic boundary.

\section{REFERENCES}

1. A. S. Besicovitch, A general form of the covering principle and relative differentiation of additive functions. II, Proc. Cambridge Philos. Soc. 42 (1946), 1-10. MR 7, 281.

2. J. R. Hattemer, Boundary behavior of temperatures. I, Studia Math. 25 (1964/65), 111-155. MR 31 \#6064.

3. R. A. Hunt and R. L. Wheeden, On the boundary values of harmonic functions, Trans. Amer. Math. Soc. 132 (1968), 307-322. MR 37 \#1634.

4. - Positive harmonic functions on Lipschitz domains, Trans. Amer. Math. Soc. 147 (1970), 507-528.

5. B. F. Jones, Jr. and C. C. Tu, Non-tangential limits for a solution of the heat equation in a two-dimensional Lip $\mathrm{p}_{\alpha}$ region, Duke Math. J. 37 (1970), 243-254. MR 41 \#4026.

6. J. T. Kemper, Kernel functions and parabolic limits for the heat equation, Thesis, Rice University, Houston, Texas, 1970.

7. - Kernel functions and parabolic limits for the heat equation, Bull. Amer. Math. Soc. 76 (1970), 1319-1320. MR 41 \#8842.

8. I. G. Petrowski, Zur Ersten Randwertaufgaben der Warmeleitungsgleichung, Compositio Math. 1 (1935), 383-419.

Courant Institute of Mathematical Sciences, New York University, New York, NEW YORK 10012 